\title{
Manejo de pacientes dependientes de opiáceos: Comparación del coste asociado al uso de buprenorfina/naloxona o metadona, y sus interacciones con tratamientos concomitantes para comorbilidades infecciosas o psiquiátricas
}

\author{
Management of opioid-dependent patients: comparison of the cost associated \\ with use of buprenorphine/naloxone or methadone, and their interactions \\ with concomitant treatments for infectious or psychiatric comorbidities
}

\begin{abstract}
Carlos Roncero*, Raquel Domínguez-Hernández**, Tomás Díaz***, José Manuel Fernández****, Rafael Forcada*****, José Manuel Martínez******, Pedro Seijo********, Antonio Terán********, Itziar Oyagüez**

* CAS Drogodependencias Vall Hebron. Hospital Universitario Vall d'Hebron-ASPB. CIBERSAM. Departamento de Psiquiatría. Universidad Autónoma de Barcelona, Barcelona. ** Pharmacoeconomics \& Outcomes Research Iberia. Madrid. ***Unidad de Tratamiento de Toxicomanías de Oviedo (UTT). Unidad de Desintoxicación Hospitalaria (UDH). Servicios de Salud Mental Área IV del Principado de Asturias. ****Unidad Asistencial de Drogodependencias de Riveira, La Coruña. *****Unidad de Conductas Adictivas de Moncada, Valencia. *******entro de Tratamiento Ambulatorio de las Adicciones de Cádiz, Diputación de Cádiz. *******Centro de Tratamiento Ambulatorio de las Adicciones de Villamartín, Diputación de Cádiz. ********Centro de Atención a Drogodependencias "San Juan de Dios", Palencia.
\end{abstract}

\section{Resumen}

El objetivo fue estimar en pacientes con dependencia a opiáceos (DO), el coste anual del manejo de interacciones del tratamiento sustitutivo con buprenorfina/ naloxona $\left(\right.$ Suboxone $\left.^{\oplus}\right) \quad(\mathrm{B} / \mathrm{N})$ o metadona, asociado con tratamientos concomitantes por comorbilidades infecciosas (VIH) o psiquiátricas. Se realizó un análisis de costes (€, 2013), del tratamiento sustitutivo y del manejo de interacciones. El coste del tratamiento de $\mathrm{B} / \mathrm{N}$ incluyó costes farmacológicos, elaboración, distribución y dispensación, en función del régimen de administración (centro asistencial o domiciliaria) y del tipo y frecuencia de dispensación (centro asistencial o farmacia), y visitas al especialista para prescripción. El coste de tratamiento con metadona incluyó, además, frascos monodosis, coste de custodia en farmacia, determinación en orina y visitas a enfermería. Se identificaron las interacciones para determinar los recursos sanitarios adicionales consumidos por la administración conjunta del tratamiento sustitutivo y concomitante (antirretrovirales, bactericidas/antifúngicos, antipsicóticos, ansiolíticos, antidepresivos y anticonvulsivos). El coste anual/paciente estimado del tratamiento sustitutivo fue de $1.525,97 €(\mathrm{~B} / \mathrm{N})$ y $1.467,29 €$ (metadona). El coste promedio anual/paciente estimado del manejo de interacciones fue de 257,07€ (infecciosas), 114,03€ (psiquiátricas) y 185,55€ (ambas) con metadona, y de 7,90€ con $\mathrm{B} / \mathrm{N}$ por comorbilidades psiquiátricas. El coste total anual/paciente estimado de $\mathrm{B} / \mathrm{N}$ fue $1.525,97 €, 1.533,87 €$ y $1.533,87 €$ comparado con $1.724,35 €, 1.581,32 €$ y $1.652,84 €$ de metadona, en pacientes que presentan comorbilidad infecciosa, psiquiátrica o ambas, respectivamente. Comparado con metadona, el coste total por paciente con DO de B/N fue menor (47,45€-198,38€ anuales) derivado de la diferencia del coste por manejo de interacciones del tratamiento concomitante de las comorbilidades infecciosas y/o psiquiátricas.

Palabras clave: análisis de costes, dependencia de opiáceos, buprenorfina/ naloxona, metadona, comorbilidades.

\begin{abstract}
The objective was to estimate the annual interaction management cost of agonist opioid treatment (AOT) for opioid-dependent (OD) patients with buprenorphine-naloxone (Suboxone $\mathrm{e}^{\circledR}$ (B/N) or methadone associated with concomitant treatments for infectious (HIV) or psychiatric comorbidities. A costs analysis model was developed to calculate the associated cost of AOT and interaction management. The AOT cost included pharmaceutical costs, drug preparation, distribution and dispensing, based on intake regimen (healthcare center or take-home) and type and frequency of dispensing (healthcare center or pharmacy), and medical visits. The cost of methadone also included single-dose bottles, monthly costs of custody at pharmacy, urine toxicology drug screenings and nursing visits. Potential interactions between AOT and concomitant treatments (antivirals, antibacterials/antifungals, antipsychotics, anxiolytics, antidepressant and anticonvulsants), were identified to determine the additional use of healthcare resources for each interaction management. The annual cost per patient of AOT was $€ 1,525.97$ for $\mathrm{B} / \mathrm{N}$ and $€ 1,467.29$ for methadone. The average annual cost per patient of interaction management was $€ 257.07$ (infectious comorbidities), €114.03 (psychiatric comorbidities) and $€ 185.55$ (double comorbidity) with methadone and $€ 7.90$ with $\mathrm{B} / \mathrm{N}$ in psychiatric comorbidities. Total annual costs of $\mathrm{B} / \mathrm{N}$ were $€ 1,525.97, € 1,533.87$ and $€ 1,533.87$ compared to $€ 1,724.35, € 1,581.32$ and $€ 1,652.84$ for methadone per patient with infectious, psychiatric or double comorbidity respectively. Compared to methadone, the total cost per patient with OD was lower with $\mathrm{B} / \mathrm{N}$ (€47.45-€198.38 per year). This is due to the differences in interaction management costs associated with the concomitant treatment of infectious and/or psychiatric comorbidities.

Key Words: costs analysis, buprenorphine-naloxone, methadone, comorbidities, opioid dependence.
\end{abstract}




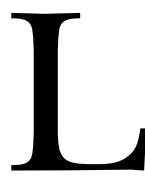

a adicción a los opioides, como la heroína, conlleva relevantes problemas médicos, sociales y económicos tanto para el individuo como para la sociedad Canadian Agency for Drugs and Technologies in Health, 2013). En la actualidad, existen diferentes terapias para combatir la dependencia a opiáceos siendo metadona y buprenorfina/naloxona $(\mathrm{B} / \mathrm{N})$ (Suboxone $^{\circledR}$ ) las alternativas terapéuticas más establecidas como tratamiento sustitutivo con agonistas opiáceos en España. En el año 2011, 76.263 personas con edades comprendidas entre 15 y 64 años, fueron tratadas en programas sustitutivos a opiáceos, de las cuales el 97,29\% fueron atendidas en programas de mantenimiento con metadona y el 2,71\% con B/N (Ministerio de Sanidad, Servicios Sociales e Igualdad, 2011).

Metadona es el agonista opioide más utilizado para la adicción a la heroína, cuya dispensación, en su mayoría, es llevada a cabo en centros asistenciales de tratamiento. $\mathrm{B} / \mathrm{N}$ en forma de comprimido y de uso sublingual, ha demostrado ser un tratamiento eficaz para los adictos a la heroína y puede ser utilizado de forma ambulatoria lo que resultaría más cómodo para los pacientes (Sittambalam, Vij y Ferguson, 2014).

Habitualmente los pacientes permanecen largos periodos en tratamiento de mantenimiento (Roncero et al., 2011), siendo la duración media de la terapia sustitutiva con opiáceos de 26 meses (Observatorio Vasco de Drogodependencias, 2004), la cual se divide normalmente en tres fases: inducción, mantenimiento y reducción del tratamiento. La fase de inducción, tiene una duración de tres días, durante los cuales se sustituye el opiáceo que el paciente ha consumido anteriormente y se ajusta la posología en función de la respuesta clínica del paciente. En la fase de mantenimiento, con una duración de meses a años, se establece una pauta posológica que evite la presencia de síntomas de abstinencia. La fase de retirada se realiza cuando el paciente alcanza una estabilidad clínica mantenida en el tiempo y suele ser iniciada con posterioridad al año de tratamiento (Terán, 2010).

Los pacientes con dependencia a opiáceos (DO) presentan una elevada comorbilidad clínica, siendo las más comunes las enfermedades infecciosas y los trastornos psiquiátricos. Las comorbilidades infecciosas más frecuentes son las relacionadas con Virus de la Inmunodeficiencia Humana (VIH), Virus de la Hepatitis C (VHC) y coinfecciones de ambos VHC/VIH con una prevalencia entre $21 \%-53 \%$, el 47 $73 \%$ y el $14 \%$ respectivamente (González-Saiz et al., 2011; Roncero et al., 2011; Sanvisens et al., 2014). La prevalencia de la comorbilidad psiquiátrica, en base a la clasificación de los trastornos psiquiátricos de acuerdo a los ejes (I, II) del DSM-IV-TD (American Psychiatric Association, 2000), se sitúa entre 25-78\% (Pereiro, Pino, Flórez, Arrojo y Becoña, 2013; Roncero et al., 2011; Sanvisens et al., 2014). Estos pacientes en su mayoría reciben tratamiento concomitante para tratar las diferentes enfermedades, siendo un 30,6\% los pacientes tratados por enfermedad infecciosa y un 21,6\% por enfermedad psiquiátrica (Roncero et al., 2011).
Es frecuente que el paciente reciba tratamiento para las enfermedades infecciosas y para los otros trastornos psiquiátricos, ya que la presencia de algunos trastornos mentales se ha asociado a mayores conductas de riesgo para desarrollar enfermedades infecciosas (Cervera, Valderrama, Bolinches, Salazar y Martínez, 1998).

La administración conjunta del tratamiento opiáceo sustitutivo y los otros fármacos puede ocasionar tanto efectos secundarios (Haro, 2012) como interacciones farmacológicas (farmacocinéticas o farmacodinámicas) que pueden determinar modificaciones en las concentraciones plasmáticas o afectar a la eficacia y seguridad de los diferentes fármacos (Sociodrogoalcohol, 2010). Para evitar posibles síntomas de abstinencia o sobredosis provocadas por la interacción, es necesario realizar ajustes en la posología y un mayor seguimiento de los pacientes (Puche, Faus, Soler y Blasco, 2000). Esto puede generar un incremento en el consumo de recursos sanitarios y por consiguiente un aumento en los costes del manejo de la enfermedad.

Las evaluaciones económicas publicadas sobre los programas de deshabituación son escasas. La mayoría se centran en los costes farmacológicos y asistenciales que supone el programa de mantenimiento con metadona (Cobacho, López y Ramos, 2011; Del Pozo, Soldevilla, Murga y Antoñanzas, 2012; Puigdollersa, Cotsa, Brugal, Torralba y Domingo-Salvany, 2003) y muy pocos en B/N (Martínez-Raga, González-Saiz, Pascual, Casado y Sabater, 2010; Martínez-Raga et al., 2012). En la actualidad, es fundamental considerar todos los costes asociados o complementarios a los distintos tratamientos que reciben los pacientes, con el objetivo de buscar los más efectivos y eficientes (Bernal-Delgado, Campillo-Artero y García-Armesto, 2014; López-Bastida et al., 2010). Sin embargo, la estimación de estos costes se hace compleja debido a las diferencias existentes entre los diversos centros asistenciales en los que pueden ser atendidos estos pacientes, por la forma de prescripción y dispensación del fármaco opiáceo, por el seguimiento del paciente según el tipo de centro entre las diferentes comunidades y por la variabilidad asociada al manejo de la comorbilidad. A pesar de la gran presencia de comorbilidad médica y de patología dual en los dependientes de opiáceos que siguen programas de mantenimiento con opiáceos (González-Saiz et al., 2011; Roncero et al., 2011; Sanvisens et al., 2014; Szerman et al., 2014), y de estudios que evalúen las interacciones producidas por la medicación concomitante, hasta la fecha no hay información disponible del coste adicional que supone el manejo de interacciones farmacológicas en la clínica diaria en este tipo de pacientes.

Este análisis pretende estimar y comparar el coste anual asociado al tratamiento sustitutivo con $\mathrm{B} / \mathrm{N}$ o metadona de los pacientes con DO junto con el coste del manejo de las posibles interacciones ocasionadas por la administración conjunta del fármaco opiáceo y la medicación por comorbilidad infecciosa y psiquiátrica, y ver si existen diferencias significativas entre ambos tratamientos. 


\section{Método}

\section{Diseño del estudio}

Se diseñó un análisis de costes en base a un árbol de decisión representando la práctica clínica (figura 1) para calcular el coste anual del tratamiento sustitutivo en un paciente con DO y el coste del manejo de las interacciones producidas por la coadministración entre fármacos en un paciente con al menos una comorbilidad infecciosa y/o psiquiátrica.

El análisis se realizó con el programa Microsoft Excel $^{\circledR}$ 2010. Se incluyeron todos los costes del tratamiento sustitutivo de las alternativas evaluadas ( $\mathrm{B} / \mathrm{N}$ y metadona) y los costes asociados a la presencia de comorbilidad infecciosa y psiquiátrica.

La identificación de los recursos sanitarios se realizó mediante un cuestionario enviado a un panel de expertos (PE) formado por un grupo de siete clínicos expertos en la atención de pacientes con DO de distintas áreas geográficas de España. El cuestionario incluyó datos obtenidos de la literatura sobre el manejo terapéutico y de la comorbilidad física y psiquiátrica y preguntas sobre aquellos datos no encontrados en la bibliografía y necesarios para la realización del estudio. Los resultados de los cuestionarios fueron unificados y depurados para llegar por parte del PE a un consenso común en el manejo clínico de los pacientes con DO en tratamiento sustitutivo y con comorbilidades asociadas, en los diferentes centros asistenciales en la práctica clínica en España.

El análisis se realizó desde la perspectiva del Sistema Nacional de Salud (SNS), siguiendo las recomendaciones nacionales e internacionales para este tipo de estudios (López-Bastida et al., 2010; Prieto et al., 2004). El horizonte temporal fue de un año, por lo que no se aplicó tasa de descuento.

Los resultados se expresaron como coste medio por paciente.

\section{Recursos y costes}

La estimación del coste total por paciente para cada una de las alternativas incluyó por un lado el coste del tratamiento sustitutivo, el cual englobó coste farmacológico, de elabo- ración, distribución y dispensación, y por otro el coste del manejo de las interacciones considerando el consumo adicional de recursos sanitarios (aumento o disminución del fármaco opiáceo, visitas a psiquiatría y a enfermería, electrocardiogramas, pruebas de determinación de niveles de metadona en sangres y en orina y frascos monodosis para la dispensación de metadona) asociado a dicho manejo en práctica clínica habitual.

Tratamiento sustitutivo. La posología empleada para la estimación del coste farmacológico del tratamiento sustitutivo, incluyó las dosis medias diarias en fase de inducción y de mantenimiento siendo $10 \mathrm{mg}$ durante 3 días y $8 \mathrm{mg}$ durante 362 días para B/N y 50,45 mg durante 14 días y 61,52 mg durante 351 días para metadona (Roncero et al., 2011). Para el cálculo del coste de B/N se consideró el Precio de Venta al Público (PVP+IVA) (Consejo General de Colegios Oficiales de Farmacéuticos, 2013). El coste farmacológico finalmente incluido en el análisis fue el del financiador (SNS). En la estimación del coste farmacológico anual de $\mathrm{B} / \mathrm{N}$ asumido por el financiador $(1.461,43 €)$ se consideró la situación laboral de los pacientes con DO (Roncero et al., 2011), así como la distribución por niveles de renta $(<18.000 €$, 18.000€-100.000€, >100.000€) en cada uno de estos grupos (Instituto Nacional de Estadística, 2013), la proporción de copago por cada tipo de paciente y el límite de aportación mensual máxima (Real Decreto-ley 16/2012) (Tabla 1).

Metadona es un fármaco que se elabora de forma centralizada y posteriormente es distribuido con medidas de seguridad a las diferentes comunidades autónomas que son las encargadas de repartirlo a los centros de dispensación y a las farmacias autorizadas (Cobacho et al., 2011). En el presente análisis, los costes asociados a la elaboración $(378,57 €)$ y distribución $(258,31 €)$ así como el coste de metadona (PVL/ $\mathrm{kg}$ ) se obtuvieron de la literatura (Martínez-Raga et al., 2012), y se actualizaron a 2013 en base a la tasa de variación del Índice de Precios de Consumo del Instituto Nacional de Estadística (Instituto Nacional de Estadística, 2011).

En la dispensación se diferenciaron dos grupos de pacientes en función de la forma de administración y de dispensación de la dosis. Para metadona, la administración

Tabla 1

Datos aplicados a la estimación de pacientes en función de las rentas y la situación laboral.

\begin{tabular}{|c|c|c|c|c|c|c|c|}
\hline GRUPO & & PENSIONISTAS & & & ACTIVOS & & EXENTOS \\
\hline Nivel de renta & $<18.000$ & $18.000-100.000$ & $>100.000$ & $<18.000$ & $18.000-100.000$ & $>100.000$ & ---- \\
\hline Roncero et al., 2013 & & $21,00 \%$ & & & $24,50 \%$ & & $52,00 \%$ \\
\hline$(\%)$ de Co-Pago & $10 \%$ & $10 \%$ & $60 \%$ & $40 \%$ & $50 \%$ & $60 \%$ & 0 \\
\hline Límite (€uro) Máximo mensual paciente & $8,14 €$ & $12,18 €$ & $61,08 €$ & $\mathrm{~N} / \mathrm{A}$ & $\mathrm{N} / \mathrm{A}$ & $\mathrm{N} / \mathrm{A}$ & $\mathrm{N} / \mathrm{A}$ \\
\hline Pacientes según rentas $(\%)$ & $84,16 \%$ & $15,57 \%$ & $0,18 \%$ & $58,19 \%$ & $40,97 \%$ & $0,84 \%$ & $100,00 \%$ \\
\hline
\end{tabular}

Nota. N/A: no aplica 


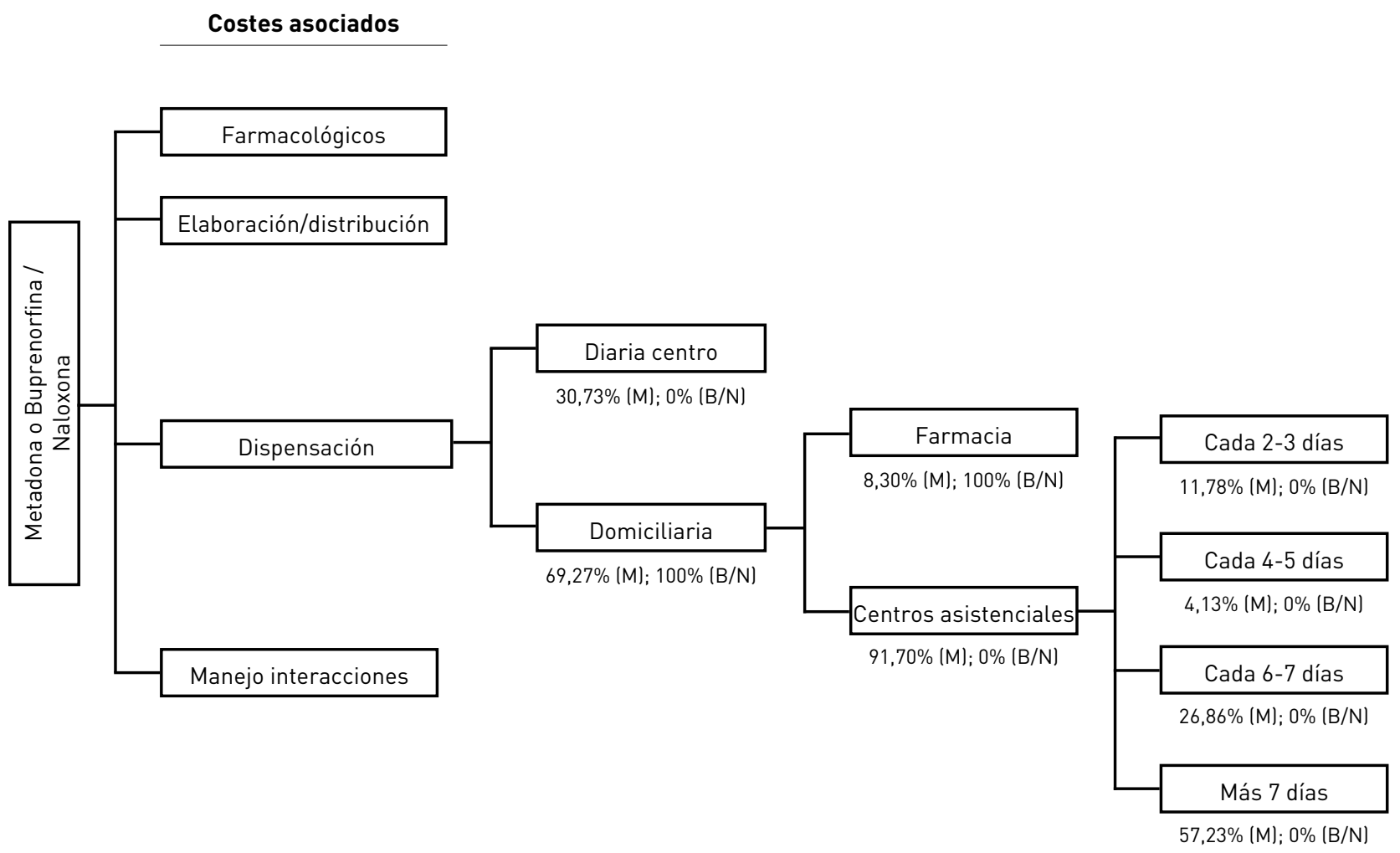

Figura 1. Diseño del estudio. Distribución de Pacientes.

se realizó diariamente en el centro asistencial o de forma domiciliaria. La dispensación para los pacientes en régimen domiciliario se hizo en el centro asistencial con una frecuencia de 2-3 días, 4-5 días, 6-7 días o más de 7 días o a través de oficina de farmacia. En el caso de B/N se asumió que la dispensación se realizó en oficina de farmacia. La distribución de los pacientes asignados a cada grupo se extrajo de un estudio observacional realizado en España (Roncero et al., 2011) (Figura 1).

Los recursos por paciente considerados para metadona fueron: 5 minutos de enfermería cuando se dispensó en el centro asistencial o la custodia del fármaco si se realizó en oficina de farmacia y un frasco monodosis diario. Además, se asumió un incremento del $50 \%$ en frascos que se asoció a los pacientes metabolizadores rápidos $(10 \%)$ a los que se les divide la dosis en varias tomas (Instituto de Adicciones Madrid, 2008). En ningún caso se consideró la reutilización del frasco monodosis. Se incluyó la realización de una prueba de metadona en orina cada 3 meses. En ambos tratamientos se incluyó una visita médica cada 6 semanas para la prescripción.

Manejo de interacciones. La asociación entre diferentes fármacos puede ocasionar interacciones farmacológicas que requieren un mayor control de los pacientes (Bruce, Moody, Altice, Gourevitch y Friedland, 2013). Es por ello que, se analizaron los costes asociados al consumo adicional de recursos en el manejo de las interacciones producidas por la administración conjunta con otros fármacos. Los grupos terapéuticos incluidos fueron antirretrovirales, bactericidas/antifúngicos, antipsicóticos, ansiolíticos, antidepresivos y anticonvulsivos (McCance-Katz, Sullivan y Nallani, 2010; McCance-Katz, 2012). A partir de datos aportados en la literatura (Amariles, Giraldo y Faus, 2007; Bruce, Altice, Gourevitch y Friedland, 2006; Gallego, Barreio y López-Ibor, 2012; McCance-Katz et al. 2010; McCance-Katz, 2012; Pérez, Jornet y Bonet, 2002; Puche et al., 2000; Serrano, 2011) y el PE se identificó de cada grupo los fármacos incluidos en el análisis por su posible interacción con $\mathrm{B} / \mathrm{N}$ o metadona y por su uso en práctica clínica habitual. A su vez, con el fin de estimar el coste que representa un paciente estándar, el panel de expertos, en base a su experiencia clínica, estimó el consumo adicional desagregado de recursos sanitarios generados por la interacción, la frecuencia y el porcentaje de pacientes que hacen uso de cada recurso para cada opción de tratamiento. En el caso de B/N, los fármacos considerados en el análisis por su potencial interacción y por la variación generada en el manejo del paciente, no siendo esta la forma habitual, fueron citalopram y escitalopram (Tabla 2). No se consideró la posible interacción entre los fármacos incluidos como medicación concomitante ni la variación de la dosis de este último. Tampoco se analizó el coste que supondría el uso de aquellos fármacos que por la gravedad de la interacción no son administrados habitualmente a los pacientes. 
Carlos Roncero, Raquel Domínguez-Hernández, Tomás Díaz, José Manuel Fernández,

Rafael Forcada, José Manuel Martínez, Pedro Seijo, Antonio Terán, Itziar Oyagüez

Tabla 2

Consumo de recursos del manejo de las interacciones por grupos terapéuticos para metadona.

\begin{tabular}{|c|c|c|c|c|c|c|c|}
\hline \multirow{2}{*}{ Fármacos } & \multirow{2}{*}{ Manejo } & \multicolumn{2}{|c|}{ Dosis (mg/día) } & \multicolumn{2}{|c|}{ Frecuencia (anual) } & \multicolumn{2}{|c|}{ Pacientes $(\%)$} \\
\hline & & Metadona & $\mathrm{B} / \mathrm{N}^{\mathrm{a}}$ & Metadona & $\mathrm{B} / \mathrm{N}^{\mathrm{a}}$ & Metadona & $\mathrm{B} / \mathrm{N}^{\mathrm{a}}$ \\
\hline \multirow{3}{*}{ citalopram/escitalopram } & Visitas psiquiatría & & & 2 & 2 & 100 & 100 \\
\hline & Electrocardiograma & & & 1 & 1 & 100 & 100 \\
\hline & & \multicolumn{2}{|c|}{ Metadona } & \multicolumn{2}{|c|}{ Metadona } & \multicolumn{2}{|c|}{ Metadona } \\
\hline \multirow{6}{*}{$\begin{array}{l}\text { efavirenz, lopinavir y } \\
\text { nevirapina }\end{array}$} & Aumento dosis & \multicolumn{2}{|c|}{24,61} & \multicolumn{2}{|c|}{351} & \multicolumn{2}{|c|}{100} \\
\hline & Visitas psiquiatría & \multicolumn{6}{|c|}{2} \\
\hline & Visitas enfermería & \multicolumn{6}{|c|}{7} \\
\hline & $\begin{array}{l}\text { Determinación niveles } \\
\text { en sangre }\end{array}$ & \multicolumn{6}{|c|}{2} \\
\hline & Electrocardiograma & \multicolumn{6}{|c|}{1} \\
\hline & Frascos & \multicolumn{6}{|c|}{365} \\
\hline \multirow{2}{*}{ indinavir } & Disminución dosis & \multirow{2}{*}{\multicolumn{2}{|c|}{6,15}} & \multicolumn{2}{|c|}{351} & \multicolumn{2}{|c|}{100} \\
\hline & Visitas psiquiatría & & & \multicolumn{2}{|l|}{1} & \multicolumn{2}{|c|}{100} \\
\hline \multirow{2}{*}{ rilpivirina } & Visitas psiquiatría & & & \multicolumn{2}{|l|}{2} & \multicolumn{2}{|c|}{100} \\
\hline & Electrocardiograma & & & \multicolumn{2}{|l|}{1} & \multicolumn{2}{|c|}{100} \\
\hline & Aumento dosis & 61,52 & & 351 & & 100 & \\
\hline & Visitas psiquiatría & & & 4 & & 100 & \\
\hline & Visitas enfermería & & & 16 & & 100 & \\
\hline rifampicina & $\begin{array}{l}\text { Determinación niveles } \\
\text { en sangre }\end{array}$ & & & 2 & & 5 & \\
\hline & Electrocardiograma & & & 1 & & 100 & \\
\hline & Frascos & & & 365 & & 75 & \\
\hline amitriptilina, clomipramina y & Visitas psiquiatría & & & 1 & & 100 & \\
\hline doxepina & Electrocardiograma & & & 1 & & 100 & \\
\hline $\begin{array}{l}\text { diazepam, alprazolam, } \\
\text { clonazepam, lorazepam, } \\
\text { midazolam, triazolam, } \\
\text { zolpidem y zopiclona }\end{array}$ & Visitas psiquiatría & & & 2 & & 100 & \\
\hline & Aumento dosis & 24,61 & & 351 & & 100 & \\
\hline & Visitas psiquiatría & & & 2 & & 100 & \\
\hline & Visitas enfermería & & & 7 & & 100 & \\
\hline carbamazepina & $\begin{array}{l}\text { Determinación niveles } \\
\text { en sangre }\end{array}$ & & & 2 & & 5 & \\
\hline & Electrocardiograma & & & 1 & & 50 & \\
\hline & Frascos & & & 365 & & 5 & \\
\hline ziprasidona y pimozida & Visitas psiquiatría & & & 2 & & 100 & \\
\hline & Electrocardiograma & & & 1 & & 100 & \\
\hline
\end{tabular}

Nota. ${ }^{a}$ Buprenorfina/Naloxona 
El aumento en la dosis del opiáceo se incluyó en caso de manifestación clínica del síndrome de abstinencia. El incremento en visitas a especialistas y a enfermería se asoció con un mayor seguimiento y una variación en la pauta de tratamiento. La prueba de determinación de niveles en sangre se asoció a dosis de metadona superiores a $100 \mathrm{mg} /$ día, a tratamientos antirretrovirales, y a fármacos susceptibles de interferir con el metabolismo de metadona (Instituto de Adicciones Madrid, 2008). El electrocardiograma se relacionó con aquellos fármacos que pueden producir una prolongación del intervalo QT y el incremento en frascos a aquellos fármacos que aumentan el metabolismo de metadona y que provocan un fraccionamiento en la dosis. Para el cálculo del coste total por comorbilidad infecciosa o psiquiátrica, se realizó un promedio de costes entre los fármacos con potencial interacción incluidos en cada comorbilidad.

Para estimar la frecuencia mensual de cada recurso se asumió que un mes equivale a 30,4 días. Todos los costes incluidos en el análisis fueron costes directos sanitarios y se expresaron en euros (€) del año 2013 (Tabla 3).

Tabla 3

Costes unitarios de fármacos y recursos sanitarios (€, 2013).

\begin{tabular}{lc}
\hline & Coste unitario \\
\hline Fármacos & $0,0006 € / \mathrm{kg}(\mathrm{PVL})$ \\
\hline Metadona & $0,50 € / \mathrm{mg}(\mathrm{PVP}+\mathrm{IVA})$ \\
\hline Buprenorfina/naloxona (Suboxone $\left.{ }^{\oplus}\right)$ & \\
\hline Recursos sanitarios & $46,22 €^{\mathrm{a}}$ \\
\hline Visitas al facultativo & $0,34 €^{\mathrm{a}}$ \\
Visitas a enfermería (coste minuto) & $69 € / \mathrm{mes}^{\mathrm{b}}$ \\
Custodia metadona por paciente & $0,45 €^{\mathrm{c}}$ \\
Frasco para la dispensación & $12,03^{\mathrm{a}}$ \\
Determinación niveles metadona en orina & $33,90 €^{\mathrm{a}}$ \\
Electrocardiograma & $115,04 €^{\mathrm{a}}$ \\
\hline Determinación de niveles plasmáticos &
\end{tabular}

Nota. ${ }^{a}$ Base de datos e-Salud. ${ }^{b}$ Convenio Comunidad de Madrid con el Colegio Oficial de Farmacéuticos de Madrid (COFM), 'Panel de expertos.

\section{Análisis de sensibilidad}

Para poder determinar el grado de estabilidad de los resultados se realizaron análisis se sensibilidad (AS) univariantes con los valores de mayor incertidumbre asociados al análisis. Las variables incluidas fueron los minutos de enfermería variando en un rango de 4-6 minutos y el coste del frasco monodosis en un $\pm 20 \%$.

\section{Resultados}

$\mathrm{El}$ tratamiento sustitutivo con $\mathrm{B} / \mathrm{N}$ supuso un coste anual por paciente de $1.525,97 €$, de los cuales el $75,77 \%$ correspondió al coste farmacológico y el $24,23 \%$ a la dispensación. El coste anual del tratamiento con metadona fue de 1.467,29€, siendo el coste farmacológico el $0,86 \%$, el $43,41 \%$ de elaboración y de distribución y el 55,73\% de dispensación (Tabla 4).

Tabla 4

Coste total anual del tratamiento con $B / N$ o metadona de un paciente con comorbilidad infecciosa o psiquiátrica (€, 2013).

\begin{tabular}{lcc}
\hline \multicolumn{1}{c}{ Tipo de coste } & B/N (Suboxone ${ }^{\circledR}$ ) & Metadona \\
\hline Tratamiento sustitutivo & $1.525,97 €$ & $1.467,29 €$ \\
\hline Farmacológicos & $1.156,25 €$ & $12,58 €$ \\
Elaboración y distribución & $0,00 €$ & $636,98 €$ \\
Dispensación & $369,72 €$ & $817,73 €$ \\
\hline Manejo interacción & & \\
\hline Infecciosa & $0,00 €$ & $257,07 €$ \\
Psiquiátrica & $7,90 €$ & $114,03 €$ \\
Ambas & $7,90 €$ & $185,55 €$ \\
\hline ToTAL ANUAL PACIENTE & $1.533,87 €$ & $1.724,35 €$ \\
\hline Comorbilidad Infecciosa & $1.525,97 €$ & $1.581,32 €$ \\
\hline Ambas comorbilidades & & \\
\hline
\end{tabular}

El coste anual por paciente del manejo de interacciones por comorbilidad infecciosa o psiquiátrica para metadona fue de 257,07€ y 114,03€. B/N generó costes de 7,90€, asociados únicamente al promedio de costes del consumo generado en el manejo de las interacciones con comorbilidad psiquiátrica. Para evitar duplicar los recursos utilizados en el manejo del paciente, el coste anual de un paciente con ambas comorbilidades se calculó realizando un promedio de costes de las dos siendo $185,55 €$ (metadona) y 7,90€ $(\mathrm{B} / \mathrm{N})$.

Finalmente, el coste anual total por paciente con DO en tratamiento sustitutivo con comorbilidad infecciosa, psiquiátrica o ambas fue de $1.525,97 €, 1.533,87 €$ y $1.533,87 €$ para $\mathrm{B} / \mathrm{N}$ y $1.724,35 €, 1.581,32 €$ y $1.625,84 €$ para metadona (Figura 2).

Los AS demostraron que una variación en los minutos de enfermería destinados a la dispensación del fármaco o en el coste del frasco monodosis de un $\pm 20 \%$, se siguen generando ahorros en un rango de 6,9€-242,54€. 


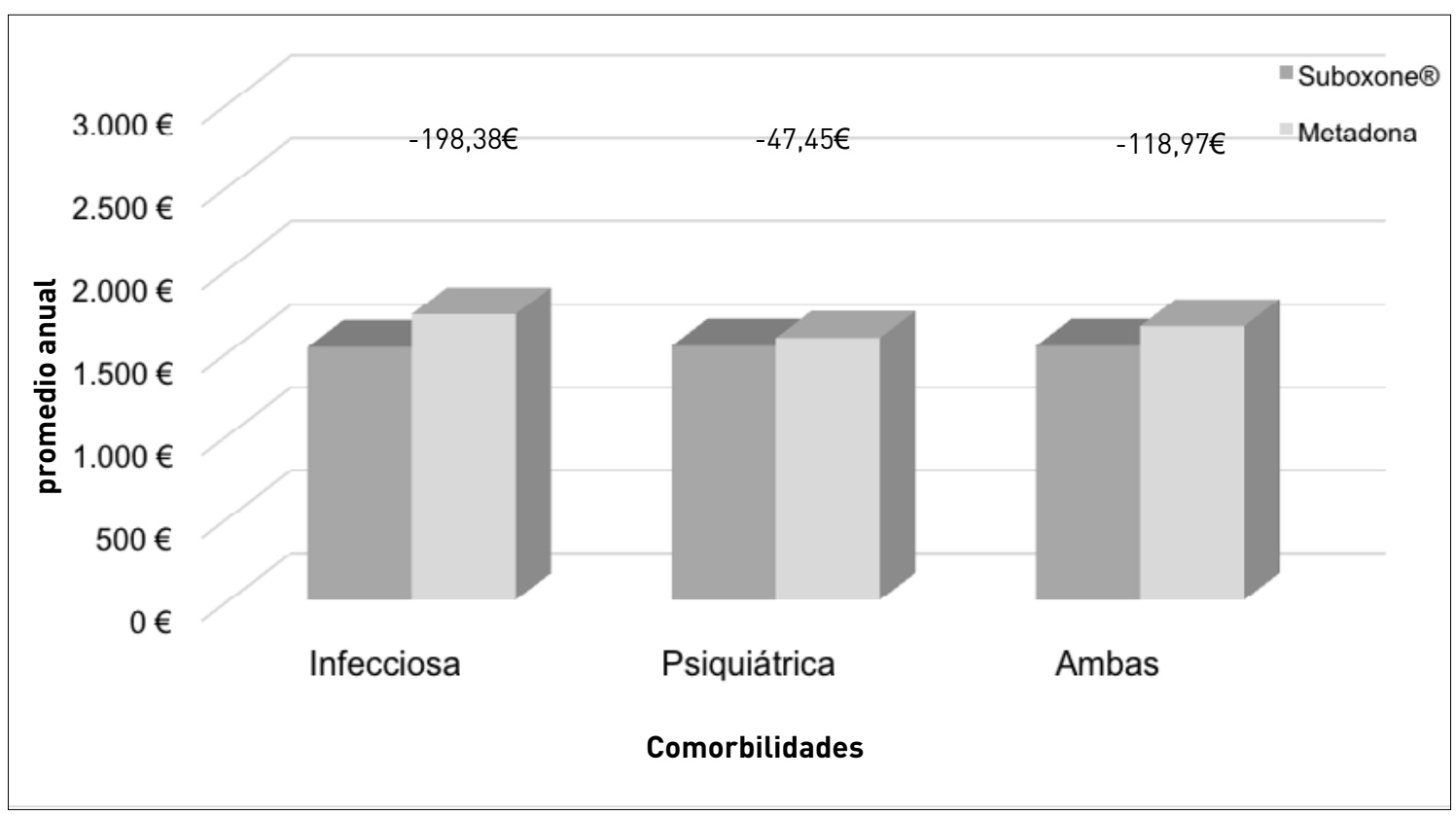

Figura 2. Resultados del análisis por paciente.

\section{Discusión}

El estudio de la presencia de comorbilidad médica o psiquiátrica en pacientes con DO es importante, debido a su elevada frecuencia de presentación (González-Saiz et al., 2011; Pereiro et al., 2013; Roncero et al., 2011), a que pueden influir en diversos aspectos de estos pacientes, como es la conducción de vehículos (Roncero et al., 2013), y a las interacciones farmacológicas producidas por la coadministración de otros fármacos, sobre todo antirretrovirales, con metadona y buprenorfina. Esto último, puede ocasionar consecuencias clínicas que hacen necesario un seguimiento más exhaustivo de los pacientes (Bruce et al., 2013) y generar una variación en el coste total del tratamiento, tal y como demuestra este trabajo.

En la actualidad es necesario la realización de planes estratégicos para optimizar los recursos existentes dedicados a los pacientes con adicciones (Ministerio de Sanidad, Servicios Sociales e Igualdad, 2013) y a los pacientes duales (Arias et al., 2013; Roncero et al., 2014; Szerman et al., 2014) y más estudios que evalúen el coste directo e indirecto que la patología supone al SNS. Por ello es necesario que además de evaluar el coste del tratamiento con $\mathrm{B} / \mathrm{N}$ o metadona, se tenga en cuenta el coste asociado a las interacciones producidas por la coadministración de fármacos, que pueden causar cambios en el seguimiento del paciente ocasionando un aumento en el consumo de recursos y por lo tanto un incremento en los costes.

En este estudio, se calcularon ambos costes, tratamiento e interacciones, para las dos alternativas dando como resultado el coste total por paciente con DO en tratamiento sustitutivo con comorbilidad infecciosa y psiquiátrica. Esta información puede resultar útil a la hora de tomar decisiones si pretendemos hacer un mejor uso de los recursos sanitarios destinados a los programas sustitutivos.

Los estudios publicados en España sobre el coste del tratamiento sustitutivo con opiáceos son escasos, casi todos se centran en el coste de los programas de mantenimiento con metadona y muy pocos comparan los costes con B/N (Martínez-Raga et al., 2010; Martínez-Raga et al., 2012). En un estudio reciente donde se comparó el impacto presupuestario de la incorporación de $\mathrm{B} / \mathrm{N}$ como tratamiento para la $\mathrm{DO}$ frente a metadona, los resultados fueron que $\mathrm{B} / \mathrm{N}$ suponía un coste adicional anual de $9 €$ (año 2007) por paciente. Los costes incluidos fueron de medicación, logísticos, dispensación, costes de personal médico y social y test toxicológico (Martínez-Raga et al., 2010). La actualización del estudio en el año 2012, el uso de B/N supuso un coste anual incremental de 10,58€ el primer año, 6,58€ el segundo y 7,34€ el tercero (costes año 2010) (Martínez-Raga et al., 2012).

A pesar de la existencia de numerosos estudios sobre las interacciones producidas por el uso de opiáceos junto con otros fármacos, en conocimiento de los autores, el presente análisis es el primer trabajo que analiza el coste que supone la comorbilidad en pacientes dependientes en la práctica clínica en España u otros países, por lo que los resultados obtenidos no pudieron ser comparados con los de otros entornos.

Es importante señalar que, el manejo de la comorbilidad psiquiátrica es muy variable y depende tanto de las características de cada paciente como de las actuaciones psicoterapéuticas llevadas a cabo simultáneamente, lo que hace que sea complejo analizar todos los costes asociados a esta comorbilidad. A su vez, este tipo de pacientes pueden ser atendidos en diferentes centros asistenciales tales como cen- 
tros de salud, centros oficiales de prescripción de opiáceos, atención primaria y en programas regionales de VIH (Roncero et al., 2011). La forma de prescripción y dispensación del fármaco y el seguimiento del paciente difiere en función del tipo de centro y de cada comunidad (Torrens, Fonseca, Castillo y Domingo-Salvany, 2013). Por ello es difícil establecer con exactitud los recursos que supone un paciente con DO. De ahí, que los resultados de los otros estudios sean tan dispersos. En el presente análisis tanto el coste asociado al tratamiento como al manejo de las interacciones, representa el consumo promedio de un paciente estándar con DO. Esto puede no ser representativo de la práctica clínica y podría influir en los resultados.

En el análisis, aunque el coste farmacológico de $\mathrm{B} / \mathrm{N}$ resultó ser mayor que metadona, al considerar los costes de elaboración, distribución y de dispensación, la diferencia disminuye significativamente. Aun así, hay que señalar que existen una serie de costes asociados a los programas de mantenimiento con metadona (amortización de los equipos, destructores de vasos, personal de seguridad en los centros, unidades móviles) que no se han considerado en este estudio (Cobacho et al., 2011; Del Pozo et al., 2012; Pellín, Gimeno, Barril, Climent y Vilanova, 2000; Puigdollersa et al., 2003). La inclusión de estos costes variables posiblemente haría incrementar el coste del tratamiento con metadona, lo que provocaría un incremento en las diferencias entre ambas estrategias evaluadas.

En el estudio hay una serie de limitaciones a tener en cuenta. La más importante es la falta de evidencia científica sobre las interacciones de $\mathrm{B} / \mathrm{N}$ con otros fármacos, por ello, se asumieron las mismas que para buprenorfina sola. La relación de fármacos que interactúan con metadona o con buprenorfina es más extensa, pero en este análisis solo se asociaron los utilizados con mayor frecuencia en la práctica clínica. Aunque hay que señalar que buprenorfina se asocia con un menor número de interacciones farmacológicas respecto a metadona (McCance-Katz, 2012; Terán, 2010), de ahí que la diferencia en los costes del manejo sea menor.

En el análisis no se tuvieron en cuenta las posibles interacciones entre los fármacos incluidos como medicación concomitante ni el coste asociado a ellas por no ser el objetivo del estudio. No se evaluó el coste de los fármacos que tienen un uso contraindicado ni los fármacos para VHC debido a que los más utilizados (interferón y ribavirina) no presentan interacción alguna con los opiáceos (Panel de expertos de Gesida, 2010). Tampoco se consideró la administración de metadona en comprimidos biselados, llevada a cabo en algunas comunidades autónomas, por no realizarse de forma habitual en la práctica clínica en España y por no disponer de datos suficientes.

Se asumió una variación de $\pm 20 \%$ en los parámetros AS (minutos de enfermería y frascos monodosis) por no disponer de más datos para el análisis.
En los análisis de costes donde se comparan más de una alternativa terapéutica, es importante que además de evaluar el coste farmacológico, se analicen los costes del manejo de los pacientes en la clínica habitual. En un estudio en el que se comparó el coste total de los pacientes con DO tratados o no con $\mathrm{B} / \mathrm{N}$, se concluyó, que aunque el coste de la medicación era mayor con $\mathrm{B} / \mathrm{N}$, al considerar los costes asociados al cuidado de estos pacientes, el coste total del tratamiento con $\mathrm{B} / \mathrm{N}$ era menor que en aquellos pacientes no tratados (Kharitonova, Aballéa, Clay, Ruby y Azh, 2014).

En este estudio se demostró que la elección de B/ $\mathrm{N}$ o metadona, conlleva implicaciones económicas cuando se trata de pacientes con comorbilidades infecciosas y psiquiátricas, ya que $\mathrm{B} / \mathrm{N}$ se asocia con un menor número de interacciones farmacológicas, lo que hace que no se produzca un incremento en el consumo de recursos ocasionado por el manejo de la interacción y como consecuencia un aumento del coste. Dada la frecuente presencia de estas comorbilidades en pacientes con DO (González-Saiz et al., 2011; Roncero et al., 2011; Sanvisens et al., 2014; Szerman et al., 2014) la elección de uno u otro fármaco podría generar ahorros importantes al SNS.

Finalmente, los resultados del presente análisis indican que, comparado con metadona, el coste total por paciente con DO de $\mathrm{B} / \mathrm{N}$ fue menor derivado de la diferencia del coste por manejo de interacciones asociadas al tratamiento concomitante de las comorbilidades infecciosas y/o psiquiátricas.

\section{Agradecimientos}

El estudio fue diseñado por Pharmacoeconomics \& Outcomes Research Iberia, una consultora especializada en Evaluación Económica de Intervenciones Sanitarias que ha recibido una financiación no condicionada de Reckitt Benckiser Pharmaceuticals para el desarrollo del análisis. Carlos Roncero, Tomás Díaz, José Manuel Fernández, Rafael Forcada, José Manuel Martínez, Pedro Seijo y Antonio Terán declaran haber recibido financiación no condicionada de Reckitt Benckiser Pharmaceuticals, por su participación para validación de la práctica clínica en España.

\section{Conflicto de intereses}

Carlos Roncero ha recibido honorarios como ponente en actividades educativas de Janssen-Cilag, Bristol-Mayers Squibb, Ferrer-Brainfarma, Pfizer, Reckitt Benckiser Pharmaceuticals, Lundbeck, Otsuka, Servier, Lilly, Shire, GSK, Rovi. También ha recibido compensación por participar como miembro en reuniones de Janssen-Cilag, Lilly, y Shire. Ha desarrollado el Proyecto PROTEUS con apoyo de una beca de Reckitt Benckiser Pharmaceuticals.

Tomás Díaz, ha participado como ponente en actividades educativas de Pfizer, Reckitt Benckiser Pharmaceuticals y Janssen-Cilag. 
José Manuel Forcada no tiene ningún interés económico por parte de otras entidades.

Rafael Forcada ha participado como ponente en actividades educativas de Janssen-Cilag y Bristol-Mayers Squibb.

José Manuel Martínez ha participado como ponente en actividades educativas de Reckitt Benckiser Pharmaceuticals, Janssen-Cilag, Bioclever 2005 SL, Pfizer, Brainpharma y Laboratorios Estévez.

Pedro Seijo ha participado como ponente en actividades educativas de Reckitt Benckinser Pharmaceuticals, Janssen-Cilag, Pfizer, Otsuka y Astrazeneca.

Antonio Terán ha recibido honorarios como ponente en actividades educativas de Janssen-Cilag, Pfizer, Lundbeck, Otsuka, Lilly, Shire, Reckitt Benckiser Pharmaceuticals; así como por la participación en reuniones de Janssen-Cilag, Lilly y Shire.

Todos los autores no tienen otras afiliaciones relevantes o intereses económicos con ninguna organización o entidad con interés económico o conflicto económico con el tema o materiales discutidos en el manuscrito, aparte de los descritos.

\section{Referencias}

Amariles, P., Giraldo, N. A. y Faus, M. J. (2007). Interacciones medicamentosas en pacientes infectados con el VIH: aproximación para establecer y evaluar su relevancia clínica. Farmacia Hospitalaria, 31, 283-302.

American Psychiatric Association. (2000). Diagnostic and Statistical Manual of Mental Disorders: DSM-IV-TR. Fourth Edition, Text Revision. Washington, D.C.: American Psychiatric Association.

Arias, F., Szerman, N., Vega, P., Mesias, B., Basurte, I., Morant, C.,... Babín. F. (2013). Estudio Madrid sobre prevalencia y características de los pacientes con patología dual en tratamiento en las redes de salud mental y de atención al drogodependiente. Adicciones, 25, 118-127.

Bernal-Delgado, E., Campillo-Artero, C. y García-Armesto, S. (2014). Oferta sanitaria y crisis: financiamos los bienes y servicios según su valor o caemos en la insolvencia. Informe SESPAS 2014. Gaceta Sanitaria, 28, 69-74. doi:10.1016/j.gaceta.2014.02.004.

Bruce, R. D., Altice, F. L., Gourevitch, M. N. y Friedland, G. H. (2006). Pharmacokinetic drug interactions between opioid agonist therapy and antiretroviral medications: implications and management for clinical practice. Journal of Acquired Immune Deficiency Syndromes, 41, 563-572.

Bruce, R. D., Moody, D. E., Altice, F.L., Gourevitch, M. N. y Friedland, G. H. (2013). A review of pharmacological interactions between HIV or hepatitis $\mathrm{C}$ virus medications and opioid agonist therapy: implications and management for clinical practice. Expert Review of Clinical Pharmacology, 6, 249-269. doi:10.1586/ecp.13.18.
Canadian Agency for Drugs and Technologies in Health. (2013). Rapid Response Reports. Suboxone ${ }^{\circledR}$ versus Methadone for the Treatment of Opioid Dependence: A Review of the Clinical and Cost-effectiveness. Recuperado de http:/ / www.ncbi.nlm.nih.gov/pubmedhealth/PMH0064785/

Cervera, G., Valderrama, J. C., Bolinches, F., Salazar, A. y Martinez, J. (1998). Variables related to risk taking behavior for HIV transmission among drug-dependent patients. Actas Luso Española de Neurología Psiquiatría y Ciencias Afines, 26, 155-164.

Cobacho, M. B., López, A. y Ramos, J. M. (2011). Análisis de costes del programa de mantenimiento de metadona en la comunidad de Murcia. Informe para la Formación e Investigación Sanitaria de la Región de Murcia. Recuperado de http:/ / www.e-drogas.es /

Consejo General de Colegios Oficiales de Farmacéuticos. (2013). Catálogo de Medicamentos. Bot Plus. Madrid. Recuperado de https://botplusweb.portalfarma.com/.

Del Pozo, J., Soldevilla, D., Murga, J. A. y Antoñanzas, F. (2012). Análisis de costes del programa de metadona de en la comunidad autónoma de la Rioja. Revista Española de Salud Pública, 86, 543-549.

Gallego, L., Barreio, P. y López-Ibor, J. J. (2012). Psychopharmacological treatments in HIV patients under antiretroviral therapy. AIDS Reviews, 14, 101-11.

González-Saiz, F., Lozano, O. M., Martín, J., Bilbao, I., Ballesta, R. y Gutierrez, J. (2011). Comorbilidad psiquiátrica en una muestra de pacientes con dependencia de opiáceos tratados con buprenorfina sublingual en régimen de comunidad terapéutica. Revista de Psiquiatría y Salud Mental (Barcelona), 4, 81-87. doi:10.1016/j. rpsm.2011.01.003.

Haro, G. (2012). New clinical implications of opiate maintenance treatments. International Journal of High Risk Behaviours $\mathcal{E}$ Addiction, 1, 88-89. doi:10.5812/ijhrba.6532.

Instituto de Adicciones Madrid. (2008). Protocolo de Metadona. Madrid: Madrid Salud Recuperado de http:/ / www.madridsalud.es/adicciones/ProtocolosSanitariosAdicciones2015.pdf

Instituto Nacional de Estadística. (2011). Cálculo de variaciones del Índice de Precios de Consumo (sistema IPC base 2011). Recuperado de http://www.ine.es/varipc/

Kharitonova, E., Aballéa, S., Clay, E., Ruby J. y Azh, V. (2014, mayo). Comparison of healthcare resource use and costs in prescription opioid-dependent patients treated with Buprenorphine/Naloxone and patients without pharmacological treatment: retrospective analysis of insurance claims in the US Public Healthcare System. Póster presentado en 11th EUROPAD conference, Glasgow, Reino Unido.

López-Bastida J., Oliva, J., Antoñanzas, F., García-Altés, A., Gisbert, R., Mar. J. y Puig-Junoy J. (2010). Propuesta de guía para la evaluación económica aplicada a las tecnologías Sanitarias. Gaceta Sanitaria, 24, 154-170. doi:10.1016/j.gaceta.2009.07.011. 
Martínez-Raga J., González-Saiz F., Pascual C., Casado M. A. y Sabater F. J. (2010). Suboxone (buprenorphine/ naloxone) as an agonist opioid treatment in Spain: a budgetary impact analysis. European Addiction Research, 16, 31-42. doi:10.1159/000259614.

Martínez-Raga, J., González-Saiz, F., Oñate, J., Oyagüez, I., Sabater, E. y Casado, M. A. (2012). Budgetary impact analysis of buprenorphe-naloxone combination (Suboxone $^{\circledR}$ ) in Spain. Health Economics Review, 2, 3. doi:10.1186/2191-1991-2-3.

McCance-Katz, E.F., Sullivan, L. E. y Nallani, S. (2010). Drug interactions of clinical importance among the opioids, methadone and buprenorphine, and other frequently prescribed medications: a review. The American Journal on Addictions, 19, 4-16. doi:10.1111/j.15210391.2009.00005.x.

McCance-Katz, E. F. (2012). Drug-Drug interactions in opioids therapy. (7th ed.). Recuperado de http://www.opioiddruginteractions.com/

Ministerio de Sanidad, Servicios Sociales e Igualdad. (2011). Plan Nacional sobre Drogas: Memoria 2011. Recuperado de http://www.pnsd.msssi.gob.es/Categoria2/ publica/pdf/memo2011.pdf

Ministerio de Sanidad, Servicios Sociales e Igualdad. (2013). Plan de Acción sobre Drogas 2013-2016. Recuperado de http://www.pnsd.msssi.gob.es/Categoria2/publica/ pdf/PLAN_ACCION_SOBRE_DROGAS2013_2016.pdf

Observatorio Vasco de Drogodependencias. (2004). Perfil del Drogodependiente que no responde a los programas de mantenimiento con metadona. País Vasco: GABIA. Recuperado de http://www.osakidetza.euskadi.net/

Panel de Experto de Gesida, Secretaría del Plan Nacional sobre el Sida (SPNS) y Asociación Española para el estudio del Hígado (AEEH). (2010). Recomendaciones de Gesida/PNS/AEEH sobre tratamiento y manejo del paciente adulto coinfectado por VIH y virus de las hepatitis A, B y C. Enfermedades Infecciosas y Microbiología Clínica, 28, 31e1-31e31.

Pellín, M. C., Gimeno, C., Barril, J., Climent, J. M. y Vilanova, E. (2000). Estudio de costes de un tratamiento de mantenimiento con metadona de bajo nivel de prestaciones. Medicina Clínica, 114, 171-173.

Pereiro, C., Pino, C., Flórez, G., Arrojo, M. y Becoña, E. (2013). Psychiatric comorbidity in patients from the addictive disorders assistance units of Galicia: The COPSIAD study. PLoS One, 8, e66451. doi:10.1371/journal. pone.0066451.

Pérez, J. C., Jornet, S. y Bonet, A. (2002). Interacciones farmacocinéticas entre metadona y antirretrovirales en pacientes infectados por el virus de la inmunodeficiencia humana. Medicina Clínica (Barcelona), 119, 224-229.

Prieto, L., Sacristán, J. A., Pinto, J. L., Badia, X., Antoñanzas, F. y del Llano, J. (2004). Análisis de costes y resul- tados en la evaluación económica de las intervenciones sanitarias. Medicina Clínica (Barcelona), 122, 423-429.

Puche, E., Faus, M., Soler, E. y Blasco, J. (2000). Optimización de los programas de mantenimiento con metadona a través del conocimiento de sus interacciones farmacológicas. Trastornos Adictivos, 2, 163-171.

Puigdollersa, E., Cotsa, F., Brugal, M. T. Torralba, L. y Domingo-Salvany, A. (2003). Programas de mantenimiento de metadona con servicios auxiliares: un estudio de coste-efectividad. Gaceta Sanitaria, 17, 123-130.

Real Decreto-ley 16/2012, de 20 de abril, de medidas urgentes para garantizar la sostenibilidad del Sistema Nacional de Salud y mejorar la calidad y seguridad de sus prestaciones. BOE, Boletín Oficial del Estado No 98, 24 de abril de 2012. Recuperado de http://www.boe.es/boe/dias/2012/04/24/ pdfs/BOE-A-2012-5403.pdf

Roncero, C., Fuste, G., Barral, C., Rodriguez-Cintas, L., Martinez-Luna, N., Eiroa-Orosa, F. J. y Casas, M. (2011). Therapeutic management and comorbidities in opiate-dependent patients undergoing a replacement therapy programme in Spain: the PROTEUS study. Heroin Addiction And Related Clinical Problems, 13, 5-16.

Roncero, C., Álvarez, J., Barral, C., Gómez-Baeza, S., Gonzalvo, B., Rodriguez-Cintas, L.,... Casas, M. (2013). Driving and legal status of Spanish opioid-dependent patients. Substance Abuse Treatment Prevention And Policy, 8, 19. doi:10.1186/1747-597X-8-19.

Roncero, C., Vega, P., Martínez-Raga, J., Barral, C., Basurte-Villamor, I., Rodriguez-Cintas, L.,... Szerman, N. (2014). Professionals' perceptions about healthcare resources for co-occuring disorders in Spain. International Journal of Mental Health Systems, 8, 35. doi:10.1186/17524458-8-35.

Sanvisens, A., Rivas, I., Faure, E., Muñoz, T., Rubio, M., Fuster, D.,... Muga, R. (2014) Características de los pacientes adictos a la heroína admitidos en un programa de tratamiento con metadona. Medicina Clínica (Barcelona), 142, 53-8. doi:10.1016/j.medcli.2012.10.023.

Serrano, J. I. (2011). Interacciones farmacológicas de los nuevos antirretrovirales. Farmacia Hospitalaria, 35, 36-43. doi:10.1016/j.farma.2010.01.018.

Sittambalam, C. D., Vij, R. y Ferguson, R. P. (2014). Buprenorphine Outpatient Outcomes Project: can Suboxone ${ }^{\circledR}$ be a viable outpatient option for heroin addiction?. Journal Community Hospital Internal Medicine Perspectives, 4. doi:10.3402/jchimp.v4.22902.

Sociodrogoalcohol. (2010). Guía para el tratamiento de la adicción a opiáceos con buprenorfina/naloxona. Guías Clínicas Sociodrogoalcohol basadas en la Evidencia Clínica. Barcelona: Sociodrogoalcohol.

Szerman, N., Vega, P., Grau-López, L., Barral, C., Basurte-Villamor, I., Mesias, B.,... Roncero, C. (2014). Dual Diagnosis Resource Needs in Spain: A National Survey 
of Professionals. Journal of Dual Diagnosis, 10, 84-90. doi: 10.1080/15504263.2014.906195.

Terán, A. (2010). Experiencia clínica en el manejo de buprenorfina/naloxona. Revista Española de Sanidad Penitenciaria, 12, 40-47.

Torrens, M., Fonseca, F., Castillo, C. y Domingo-Salvany, A. (2013). Methadone maintenance treatment in Spain: the success of a harm reduction approach. Bull World Health Organ, 91, 136-141. doi:10.2471/BLT.12.111054. 